\title{
Irreducibility criterion for quasi-ordinary polynomials *
}

\author{
Abdallah Assi ${ }^{\dagger}$
}

\section{Introduction}

Let $\mathbf{K}$ be an algebraically closed field of characteristic zero, and let $\mathbf{R}=\mathbf{K}\left[\left[x_{1}, \ldots, x_{e}\right]\right]=\mathbf{K}[[\underline{x}]]$ be the ring of formal power series in $x_{1}, \ldots, x_{e}$ over $\mathbf{K}$. Let $f=y^{n}+a_{1}(\underline{x}) y^{n-1}+\ldots+a_{n}(\underline{x})$ be a nonzero polynomial of $\mathbf{R}[y]$, and suppose that $f$ is irreducible in $\mathbf{R}[y]$. Suppose that $e=1$ and let $g$ be a nonzero polynomial of $\mathbf{R}[y]$, then define the intersection multiplicity of $f$ with $g$, denoted int $(f, g)$, to be the $x$-order of the $y$ resultant of $f$ and $g$. The set of $\operatorname{int}(f, g), g \in \mathbf{R}[y]$, defines a semigroup, denoted $\Gamma(f)$. It is will known that a set of generators of $\Gamma(f)$ can be computed from polynomials having the maximal contact with $f$ (see [1] and [6]), namely, there exist $g_{1}, \ldots, g_{h}$ such that $n, \operatorname{int}\left(f, g_{1}\right), \ldots, \operatorname{int}\left(f, g_{h}\right)$ generate $\Gamma(f)$ and for all $1 \leq k \leq h$, the Newton-Puiseux expansion of $g_{k}$ coincides with that of $f$ until a characteristic exponent of $f$. In [1], Abhyankar introduced a special set of polynomials called the approximate roots of $f$. These polynomials have the advantage that they can be calculated from the equation of $f$ by using the Tschirnhausen transform. Suppose that $e \geq 2$ and that the discriminant of $f$ is of the form $x_{1}^{N_{1}} \ldots \ldots x_{e}^{N_{e}} \cdot u\left(x_{1}, \ldots, x_{e}\right)$, where $u$ is a unit in $\mathbf{K}[[\underline{x}]]$ (such a polynomial is called quasi-ordinary polynomial). By Abhyankar-Jung Theorem, the roots of $f\left(x_{1}, \ldots, x_{e}, y\right)=0$ are all in $\mathbf{K}\left[\left[x_{1}^{\frac{1}{n}}, \ldots, x_{e}^{\frac{1}{n}}\right]\right]$, in particular there exists a power series $y\left(t_{1}, \ldots, t_{e}\right)=\sum_{p} c_{p} t_{1}^{p_{1}} \ldots . t_{e}^{p_{e}} \in \mathbf{K}\left[\left[t_{1}, \ldots, t_{e}\right]\right]$ such that $f\left(t_{1}^{n}, \ldots, t_{e}^{n}, y\left(t_{1}, \ldots, t_{e}\right)\right)=0$ and the other roots of $f\left(t_{1}^{n}, \ldots, t_{e}^{n}, y\right)=0$ are the conjugates of $y\left(t_{1}, \ldots, t_{e}\right)$ with respect to the $n$th roots of unity in $\mathbf{K}$. Given a polynomial $g$ of $\mathbf{R}[y]$, we define the order of $g$ to be the leading exponent with respect to the lexicographical order of the smallest homogeneous component of $g\left(t_{1}^{n}, \ldots, t_{e}^{n}, y\left(t_{1}, \ldots, t_{e}\right)\right)$. The set of orders of polynomials of $\mathbf{R}[y]$ defines a semigroup. In this paper we first prove that the canonical basis of $(n \mathbf{Z})^{e}$ with the set of orders of the approximate roots of $f$ generate the semigroup of $f$, then we give, using these approximate roots and the notion of generalized Newton polygons, a criterion for a quasi-ordinary polynomial to be irreducible. Note that if $e=1$, then $f$ is quasi-ordinary, in particular our results generalize those of Abhyankar (see [1] and [3]).

The paper is organized as follows: in Section 1 we introduce the notion of approximate roots of a polynomial in one variable over a commutative ring with unity. In Section 2 we show how to associate a semigroup with an irreducible quasi-ordinary polynomial of $\mathbf{R}[y]$. In Section 3 we introduce the notion of pseudo roots of a quasi-ordinary polynomial $f$ then we prove that the orders of these polynomials together with the canonical basis of $(n \mathbf{Z})^{e}$ give a set of generators of the semigroup of $f$. This result remains true if we replace the pseudo roots of $f$ by its set of approximate roots. This is what we prove in Section 4 . Sections 5 and 6 are devoted to the irreducibility criterion: in Section 5 we introduce the notion of generalized Newton polygon, and we define the notion of straightness of a polynomial with respect to a set of polynomials, then we use these notions in section 6 in order to decide if a given quasi-ordinary polynomial is irreducible. We end the paper with some examples in section 7 .

*2000 Mathematical Subject Classification: 32S25, 32S70.

During the development of this work, the author visited the Department of Mathematics at the American University of Beirut, Lebanon. He would like to thank that institution for hospitality and support. He also would like to think the Center for Advanced Mathematical Sciences-CAMS for offering access to many facilities.

†Université d'Angers, Mathématiques, 49045 Angers cedex 01, France, e-mail:assi@univ-angers.fr 


\section{G-adic expansions}

Let $\mathbf{R}$ be a commutative ring with unity and let $\mathbf{R}[y]$ be the ring of polynomials in $y$ with coefficients in $\mathbf{R}$. Let $f=y^{n}+a_{1} y^{n-1}+\ldots+a_{n}$ be a monic polynomial of $\mathbf{R}[y]$ of degree $n>0$ in $y$. Let $d \in \mathbf{N}$ and suppose that $d$ divides $n$. Let $g$ be a monic polynomial in $\mathbf{R}[y]$ of degree $\frac{n}{d}$ in $y$. There exist unique polynomials $a_{1}(y), \ldots, a_{d}(y) \in \mathbf{R}[y]$ such that:

$$
f=g^{d}+\sum_{i=1}^{d} a_{i}(y) \cdot g^{d-i}
$$

and for all $1 \leq i \leq d$, if we denote by $\operatorname{deg}_{y}$ the $y$-degree, then $\operatorname{deg}_{y}\left(a_{i}\right)<\frac{n}{d}=\operatorname{deg}_{y} g$. The equation above is called the $g$-adic expansion of $f$.

This construction can be generalized to a sequence of polynomials. Let to this end $n=d_{1}>d_{2}>\ldots>d_{h}$ be a sequence of integers such that $d_{i+1}$ divides $d_{i}$ for all $1 \leq i \leq h-1$, and set $e_{i}=\frac{d_{i}}{d_{i+1}}, 1 \leq i \leq h-1$ and $e_{h}=+\infty$. For all $1 \leq i \leq h$, let $g_{i}$ be a monic polynomial of $\mathbf{R}[y]$ of degree $\frac{n}{d_{i}}$ in $y$. Set $G=\left(g_{1}, \ldots, g_{h}\right)$ and let $B=\left\{\left(\theta_{1}, \ldots, \theta_{h}\right) \in \mathbf{N}^{h}, 0 \leq \theta_{i}<e_{i}\right.$ for all $\left.1 \leq i \leq h\right\}$. Then $f$ can be uniquely written in the following form:

$$
f=\sum_{\underline{\theta} \in B} a_{\underline{\theta}} \cdot g^{\underline{\theta}}
$$

where if $\underline{\theta}=\left(\theta_{1}, \ldots, \theta_{h}\right)$, then $g^{\underline{\theta}}=g_{1}^{\theta_{1}} \ldots . g_{h}^{\theta_{h}}$ and $a_{\underline{\theta}} \in \mathbf{R}$. We call this expansion the $G$-adic expansion of $f$. We set $\operatorname{Supp}_{G}(f)=\left\{\underline{\theta} ; a_{\underline{\theta}} \neq 0\right\}$ and we call it the $G$-support of $f$.

Let $f, g$ be as above and let $f=g^{d}+\sum_{i=1}^{d} a_{i} \cdot g^{d-i}$ be the $g$-adic expansion of $f$. Assume that $d$ is a unit in $\mathbf{R}$. The Tschirnhausen transform of $f$ with respect to $g$, denoted $\tau_{f}(g)$, is defined by $\tau_{f}(g)=g+d^{-1} a_{1}$. Note that $\tau_{f}(g)=g$ if and only if $a_{1}=0$. By [1], $\tau_{f}(g)=g$ if and only if $\operatorname{deg}_{y}\left(f-g^{d}\right)<n-\frac{n}{d}$. If one of these equivalent conditions is verified, then the polynomial $g$ is called a $d$-th approximate root of $f$. By [1], there exists a unique $d$-th approximate root of $f$. We denote it by $\operatorname{App}_{y}^{d}(f)$.

\section{The semigroup of a quasi-ordinary polynomial}

Let $\mathbf{K}$ be an algebraically closed field of characteristic zero, and let $\mathbf{R}=\mathbf{K}\left[\left[x_{1}, \ldots, x_{e}\right]\right]$ (denoted $\mathbf{K}[[\underline{x}]]$ ) be the ring of formal power series in $x_{1}, \ldots, x_{e}$ over $\mathbf{K}$. Let $f=y^{n}+a_{1}(\underline{x}) y^{n-1}+\ldots+a_{n}(\underline{x})$ be a nonzero polynomial of $\mathbf{R}[y]$. Suppose that the discriminant of $f$ is of the form $x_{1}^{N_{1}} \ldots \ldots x_{e}^{N_{e}} \cdot u\left(x_{1}, \ldots, x_{e}\right)$, where $N_{1}, \ldots, N_{e} \in \mathbf{N}$ and $u(\underline{x})$ is a unit in $\mathbf{K}[[\underline{x}]]$. We call $f$ a quasi-ordinary polynomial. It follows from Abhyankar-Jung Theorem that there exists a power series $y(\underline{t})=y\left(t_{1}, \ldots, t_{e}\right) \in \mathbf{K}\left[\left[t_{1}, \ldots, t_{e}\right]\right]$ (denoted $\left.\mathbf{K}[[\underline{t}]]\right)$ such that $f\left(t_{1}^{n}, \ldots, t_{e}^{n}, y(\underline{t})\right)=0$. Furthermore, if $f$ is an irreducible polynomial, then we have:

$$
f\left(t_{1}^{n}, \ldots, t_{e}^{n}, y\right)=\prod_{i=1}^{n}\left(y-y\left(w_{1}^{i} t_{1}, \ldots, w_{e}^{i} t_{e}\right)\right)
$$

where $\left(w_{1}^{i}, \ldots, w_{e}^{i}\right)_{1 \leq i \leq n}$ are distinct elements of $\left(U_{n}\right)^{e}, U_{n}$ being the group of $n$th roots of unity in $\mathbf{K}$.

Suppose that $f$ is irreducible and let $y(\underline{t})$ be as above. Write $y(\underline{t})=\sum_{p} c_{p} \underline{t}^{p}$ and define the support of $y$ to be the set $\left\{p \mid c_{p} \neq 0\right\}$. Obviously the support of $y\left(w_{1} t_{1}, \ldots, w_{e} t_{e}\right)$ does not depend on $w_{1}, \ldots, w_{e} \in U_{n}$. We denote 
it by $\operatorname{Supp}(f)$ and we call it the support of $f$. It is well known that there exists a finite sequence of elements in $\operatorname{Supp}(f)$, denoted $m_{1}, \ldots, m_{h}$, such that

i) $m_{1}<m_{2}<\ldots<m_{h}$, where $<$ means $<$ coordinate-wise.

ii) If $c_{p} \neq 0$, then $p \in(n \mathbf{Z})^{e}+\sum_{\left|m_{i}\right| \leq|p|} m_{i} \mathbf{Z}$.

iii) $m_{i} \notin(n \mathbf{Z})^{e}+\sum_{j<i} m_{j} \mathbf{Z}$ for all $i=1, \ldots, h$.

The set of elements of this sequence is called the set of characteristic exponents of $f$. We denote by convention $m_{h+1}=(+\infty, \ldots,+\infty)$. If $e=1$, this set is nothing but the set of Newton-Puiseux exponents of $f$.

Let $u=\sum_{p} c_{p} \underline{t}^{p}$ in $\mathbf{K}[[\underline{t}]]$ be a nonzero power series. We denote by $\operatorname{In}(u)$ the initial form of $u$ : if $u=u_{d}+u_{d+1}+\ldots$ denotes the decomposition of $u$ into sum of homogeneous components, then $\operatorname{In}(u)=u_{d}$. We set $O_{t}(u)=d$ and we call it the $\underline{t}$-order of $u$. We denote by $\exp (u)$ the greatest exponent of $\operatorname{In}(u)$ with respect to the lexicographical order. We denote by $\operatorname{inco}(u)$ the coefficient $c_{\exp }(u)$, and we call it the initial coefficient of $u$. We set $\mathrm{M}(u)=\operatorname{inco}(u) \underline{t}^{\exp (u)}$, and we call it the initial monomial of $u$.

Let $g$ be a nonzero quasi-ordinary element of $\mathbf{R}[y]$. The order of $g$ with respect to $f$, denoted $O(f, g)$, is defined to be $\exp \left(g\left(t_{1}^{n}, \ldots, t_{e}^{n}, y(\underline{t})\right)\right.$. Note that it does not depend on the choice of the root $y(\underline{t})$ of $f\left(t_{1}^{n}, \ldots, t_{e}^{n}, y\right)=0$. The set $\{O(f, g) \mid g \in \mathbf{R}\}$ defines a subsemigroup of $\mathbf{Z}^{e}$. We call it the semigroup associated with $f$ and we denote it by $\Gamma(f)$.

Let $M(e, e)$ be the unit $(e, e)$ matrix. Let $D_{1}=n^{e}$ and for all $1 \leq i \leq h$, let $D_{i+1}$ be the gcd of the $(e, e)$ minors of the matrix $\left(n M(e, e), m_{1}{ }^{T}, \ldots, m_{i}{ }^{T}\right)$ (where $T$ denotes the transpose of a matrix). Since $m_{i} \notin(n \mathbf{Z})^{e}+\sum_{j<i} m_{j} \mathbf{Z}$ for all $1 \leq i \leq h$, then $D_{i+1}<D_{i}$. We define the sequence $\left(e_{i}\right)_{1 \leq i \leq h}$ to be $e_{i}=\frac{D_{i}}{D_{i+1}}$ for all $1 \leq i \leq h$.

Let $M_{0}=(n \mathbf{Z})^{e}$ and let $M_{i}=(n \mathbf{Z})^{e}+\sum_{j=1}^{i} m_{j} \mathbf{Z}$ for all $1 \leq i \leq h$. Then $e_{i}$ is the index of the lattice $M_{i-1}$ in $M_{i}$, and $n=e_{1} \ldots e_{h}$, in particular $D_{h+1}=n^{e-1}$. We set $d_{i}=\frac{D_{i}}{D_{h+1}}$ for all $1 \leq i \leq h+1$. In particular $d_{1}=n$ and $d_{h+1}=1$. The sequence $\left(d_{1}, d_{2}, \ldots, d_{h+1}\right)$ is called the gcd-sequence associated with $f$. We also define the sequence $\left(r_{k}\right)_{1 \leq k \leq h}$ by $r_{1}=m_{1}$ and $r_{k+1}=e_{k} r_{k}+m_{k+1}-m_{k}$ for all $1 \leq k \leq h-1$.

Denote by $\operatorname{Root}(f)$ the set of $n$ roots of $f\left(t_{1}^{n}, \ldots, t_{e}^{n}, y\right)=0$ introduced above and let $y(\underline{t})$ be an element of this set. We have the following:

Lemma 2.1 i) $\operatorname{In}(y(\underline{t})-z(\underline{t}))$ is a monomial for all $z(\underline{t}) \in \operatorname{Root}(f)-\{y(\underline{t})\}$. Furthermore, $\{\exp (y(\underline{t})-z(\underline{t})) \mid z(\underline{t}) \in$ $\operatorname{Root}(f)-\{y(\underline{t})\}\}=\left\{m_{1}, \ldots, m_{h}\right\}$.

ii) Let for all $1 \leq k \leq h$,

$$
\begin{aligned}
& S(k)=\left\{z(\underline{t}) \in \operatorname{Root}(f) \mid \exp (y(\underline{t})-z(\underline{t}))=m_{k}\right\} . \\
& R(k)=\left\{z(\underline{t}) \in \operatorname{Root}(f) \mid \exp (y(\underline{t})-z(\underline{t})) \geq m_{k}\right\} . \\
& Q(k)=\left\{z(\underline{t}) \in \operatorname{Root}(f) \mid \exp (y(\underline{t})-z(\underline{t}))<m_{k}\right\} .
\end{aligned}
$$

Then the cardinality of $S(k)$ (resp. $R(k)$, resp. $Q(k))$ is $d_{k}-d_{k+1}$. (resp. $d_{k}$, resp. $\left.n-d_{k}\right)$.

Proof. The proof is the same as in the case of plane curves. Note that given $z(\underline{t}) \in \operatorname{Root}(f)$, since $y(\underline{t})-z(\underline{t})$ divides the discriminant, then $y(\underline{t})-z(\underline{t})=a \cdot \underline{m}^{m} \cdot u$, where $a \in \mathbf{K}^{*}, m$ is a characteristic exponent of $f$, and $u$ is a unit in $\mathbf{K}[[\underline{t}]]$. In particular, $\operatorname{In}(y(\underline{t})-z(\underline{t}))=a \cdot \underline{t}^{m}$. 
Let $\phi(\underline{t})=\left(t_{1}^{p}, \ldots, t_{e}^{p}, Y(\underline{t})\right)$ and $\psi(\underline{t})=\left(t_{1}^{q}, \ldots, t_{e}^{q}, Z(\underline{t})\right)$ be two nonzero elements of $\mathbf{K}[[\underline{t}]]^{e+1}$. We define the contact between $\phi$ and $\psi$ to be the element $\frac{1}{p q} \exp \left(Y\left(t_{1}^{q}, \ldots, t_{e}^{q}\right)-Z\left(t_{1}^{p}, \ldots, t_{e}^{p}\right)\right)$. We denote it by c $(\phi, \psi)$.

We define the contact between $f$ and $\phi$, denoted $c(f, \phi)$, to be the maximal element in the set of contacts between $\phi$ with the roots of $f\left(t_{1}^{n}, \ldots, t_{e}^{n}, y\right)=0$.

Let $g=y^{m}+b_{1}(\underline{x}) y^{m-1}+\ldots+b_{m}(\underline{x})$ be a nonzero polynomial of $\mathbf{R}[y]$. Suppose that $g$ is an irreducible quasi-ordinary polynomial and let $\psi(\underline{t})=\left(t_{1}^{m}, \ldots, t_{e}^{m}, Z(\underline{t})\right)$ be a root of $g\left(x_{1}, \ldots, x_{e}, y\right)=0$. We define the contact between $f$ and $g$, denoted $\mathrm{c}(f, g)$, to be the contact between $f$ and $\psi$, and we recall that this definition does not depend on the choice of the root $\psi$ of $g$. Note that if $f . g$ is a quasi-ordinary polynomial, then $\operatorname{In}(f(\psi(\underline{t}))=M(f(\psi(\underline{t}))$.

With these notations we have the following proposition:

Proposition 2.2 Let $g=y^{m}+b_{1}(\underline{x}) y^{m-1}+\ldots+b_{m}(\underline{x})$ be an irreducible quasi-ordinary polynomial of $\mathbf{R}[y]$ and suppose that $f . g$ is a quasi-ordinary polynomial. Let $\left(D_{j}^{\prime}\right)_{1 \leq j \leq h^{\prime}+1}\left(\operatorname{resp} .\left(d_{j}^{\prime}\right)_{1 \leq j \leq h^{\prime}+1},\left(m_{j}^{\prime}\right)_{1 \leq j \leq h^{\prime}}\right)$ be the set of characteristic sequences associated with $g$. If $c$ denotes the contact $\mathrm{c}(f, g)$, then we have the following:

i) If for all $1 \leq q \leq h, n c \notin M_{q}$, then $O(f, g)=$ n.m.c.

ii) Otherwsie, let $1 \leq q \leq h$ be the smallest integer such that $n c \in M_{q}$, then $O(f, g)=\left(r_{q} d_{q}+(n c-\right.$ $\left.\left.m_{q}\right) d_{q+1}\right) \cdot \frac{m}{n}$.

iii) If $n c \in M_{q}-M_{q-1}$ and $n c \neq m_{q}$, then $\frac{n}{d_{q+1}} \mid m$.

Proof. i) and ii) are obvious. To prove iii) let $\phi=\left(t_{1}^{n}, \ldots, t_{e}^{m}, Y(\underline{t})\right)\left(\right.$ resp. $\left.\psi=\left(t_{1}^{m}, \ldots, t_{e}^{m}, Z(\underline{t})\right)\right)$ be a root of $f(\underline{x}, y)=0$ (resp. $g(\underline{x}, y)=0$ ) and remark that if $n c \in M_{q}-M_{q-1}$ and $n c \neq m_{q}$ then the exponents of $Z\left(t_{1}^{n}, \ldots, t_{e}{ }^{n}\right)$ coincide with those of $Y\left(t_{1}{ }^{m}, \ldots, t_{e}{ }^{m}\right)$ till at least $m_{q} . m$. Write $Y(\underline{t})=\sum_{i} c_{i} \underline{t}^{i}$ and $Z(\underline{t})=\sum_{j} c_{j}^{\prime} \underline{t}^{j}$, then for all $i \in M_{q+1}$ in $\operatorname{Supp}(Y)$, there exists $j \in \operatorname{Supp}(Z)$ such that $i . m=j . n$. But the gcd of minors of the matrix $\left(m . n M(e, e), t_{m . m_{1}}, \ldots, t_{m . m_{q}}\right)$ is $m^{e} \cdot D_{q+1}$, and the gcd of minors of the matrix $\left(m . n M(e, e), t_{n . m_{1}^{\prime}}, \ldots, t_{n . m_{q}^{\prime}}\right)$ is $n^{e} . D_{q+1}^{\prime}$. Thus $m^{e} \cdot D_{q+1}=n^{e} \cdot D_{q+1}^{\prime}$, in particular $m^{e} \cdot n^{e-1} d_{q+1}=$ $n^{e} \cdot m^{e-1} \cdot d_{q+1}^{\prime}$. This implies that $m=\frac{n}{d_{q+1}} \cdot d_{q+1}^{\prime}$, which proves our assertion.

\section{$3 \quad$ Pseudo roots and generators of the semigroup}

Let the notations be as in section 2 and let $q \in \mathbf{N}, 1 \leq q \leq h+1$. Let $y(\underline{t})=\sum c_{p} \underline{t}^{p} \in \operatorname{Root}(f)$ and consider the truncation $\bar{y}(\underline{t})=\sum_{p \in M_{q}} c_{p} \cdot \underline{t}^{p}$ of $y$. Let $G_{q}(\underline{x}, y) \in \mathbf{R}[y]$ be the minimal polynomial of $\bar{y}\left(\underline{x}^{\frac{1}{n}}\right)$ over $\mathbf{K}((\underline{x}))$. Then $G_{q}$ is a quasi-ordinary polynomial of degree $\frac{n}{d_{q}}$ in $y$, and $G_{q}\left(t_{1}^{\frac{n}{d_{q}}}, \ldots, t_{e}^{\frac{n}{d_{q}}}, \bar{y}\left(\underline{t}^{\frac{1}{d_{q}}}\right)\right)=0$. Furthermore, there exist $\frac{n}{d_{q}}$ distinct elements $\left(\rho_{1}^{i}, \ldots, \rho_{\frac{n}{d_{q}}}^{i}\right)_{1 \leq i \leq \frac{n}{d_{q}}}$ in $\left(U_{\frac{n}{d_{q}}}\right)^{e}$, where $U_{\frac{n}{d_{q}}}$ denotes the set of $\frac{n}{d_{q}}$ th roots of unity in K, such that:

$$
G\left(t_{1}^{\frac{n}{d_{q}}}, \ldots, t_{e}^{\frac{n}{d_{q}}}, y\right)=\prod_{i=1}^{\frac{n}{d_{q}}}\left(y-\bar{y}\left(\rho_{1}^{i} t_{1}^{\frac{1}{d_{q}}}, \ldots, \rho_{\frac{n}{d_{q}}}^{i} t_{e^{\frac{1}{d_{q}}}}\right)\right)
$$

We call $G_{q}$ a $d_{q}$ th pseudo root of $f$. With the notations of Section $2, c\left(f, G_{q}\right)=m_{q}$, and consequently by Proposition 2.2. ii), $O\left(f, G_{q}\right)=r_{q}$.

Let $G=\left(G_{1}, \ldots, G_{h}, G_{h+1}\right)$ be a set of $d_{k}$ th pseudo roots of $f, 1 \leq k \leq h+1$, and recall that $\operatorname{deg}_{y}\left(G_{1}\right)=1$ and that $G_{h+1}=f$. Let $B(G)=\left\{\underline{\theta} \in \mathbf{N}^{h+1} ; 0 \leq \theta_{k}<e_{k}\right.$ for all $1 \leq k \leq h$ and $\left.\theta_{h+1}<+\infty\right\}$. Given two elements 
$\underline{\theta}^{1}, \underline{\theta}^{2} \in B(G)$, and two elements $\underline{\gamma}^{1}, \underline{\gamma}^{2} \in \mathbf{N}^{e}$, if $\theta_{h+1}^{1}=\theta_{h+1}^{2}$ and $\underline{\theta}^{1} \neq \underline{\theta}^{2}$ then $\sum_{i=1}^{e} \gamma_{i}^{1} \cdot r_{0}^{i}+\sum_{k=1}^{h} \theta_{k}^{1} r_{k} \neq$ $\sum_{i=1}^{e} \gamma_{i}^{2} \cdot r_{0}^{i}+\sum_{k=1}^{h} \theta_{k}^{2} r_{k}$.

Let $F(\underline{x}, y)$ be a monic polynomial of $\mathbf{R}[y]$ and let:

$$
F=\sum_{\underline{\theta} \in B(G)} c_{\theta}(\underline{x}) G_{1}^{\theta_{1}} \ldots G_{h}^{\theta_{h}} \cdot G_{h+1}^{\theta_{h+1}}
$$

be the $G$-adic expansion of $F$. Let $\operatorname{Supp}_{G}(F)=\left\{\underline{\theta} \in B(G), c_{\theta} \neq 0\right\}$ and let $B^{\prime}(G)=\left\{\underline{\theta} \in \operatorname{Supp}_{G}(F) ; \theta_{h+1}=0\right\}$. Clearly $f$ divides $F$ if and only if $B^{\prime}(G)=\emptyset$. Otherwise, there is a unique $\underline{\theta}^{0} \in \operatorname{Supp}_{G}(F)$ such that $O(f, F)=$ $O\left(f, c_{\underline{\theta}}(\underline{x}) G_{1}^{\theta_{1}} \ldots \ldots G_{h}^{\theta_{h}}\right)=O\left(f, c_{\underline{\theta}}(\underline{x})\right)+\sum_{i=1}^{h} \theta_{i} r_{i}$. In particular, $r_{0}^{1}, \ldots, r_{0}^{e}, r_{1}, \ldots, r_{h}$ generate $\Gamma(f)$.

\section{Approximate roots of a quasi-ordinary polynomial}

Let the notations be as in Section 2, and let $y(\underline{t})=\sum_{p} c_{p} \underline{t}^{p} \in \operatorname{Root}(f)$. Given $1 \leq q \leq h$ and $z(\underline{t}) \in \operatorname{Root}(f)$, there exists $w(z) \in U_{n}$ such that the coefficient of $t^{m_{q}}$ in the expansion of $z(\underline{t})$ is $w(z) \cdot c_{m_{q}}$. Let $Q(q)$ (resp. $R(q)$, resp. $S(q))$ be the set of elements of $\operatorname{Root}(f)$ whose contact with $y(\underline{t})$ is $<m_{q}\left(\right.$ resp. $\geq m_{q}$, resp. $\left.=m_{q}\right)$ and let $\zeta$ be an element of $\mathbf{K}$. It follows from Lemma 2.1. that:

$$
\prod_{z(\underline{t}) \in R(q)}\left(\zeta-w(z) \cdot c_{m_{q}}\right)=\left(\zeta^{e_{q}}-c_{m_{q}}^{e_{q}}\right)^{d_{q+1}}
$$

On the other hand, if $q \geq 2$, since:

$$
\prod_{z(\underline{t}) \in Q(q)}(y(\underline{t})-z(\underline{t}))=\prod_{k=1}^{q-1} \prod_{z(\underline{t}) \in S(k)}(y(\underline{t})-z(\underline{t}))
$$

then

$$
\begin{aligned}
& \exp \left(\prod_{z(\underline{\underline{t}}) \in Q(q)}(y(\underline{t})-z(\underline{t}))\right)=\sum_{k=1}^{q-1} \exp \left(\prod_{z(\underline{t}) \in S(k)}(y(\underline{t})-z(\underline{t}))\right) \\
= & \sum_{k=1}^{q-1}\left(d_{k}-d_{k+1}\right) \cdot m_{k}=m_{1} d_{1}+\sum_{k=1}^{q-2}\left(m_{k+1}-m_{k}\right) d_{k+1}-m_{q-1} d_{q} \\
= & r_{1} d_{1}+\sum_{k=1}^{q-2}\left(r_{k+1} d_{k+1}-r_{k} d_{k}\right)-m_{q-1} d_{q}=r_{q-1} \cdot d_{q-1}-m_{q-1} \cdot d_{q}
\end{aligned}
$$

Consequently

$$
\exp \left(\prod_{z(\underline{t}) \in Q(q)}(y(\underline{t})-z(\underline{t}))\right)=\left\{\begin{array}{lc}
r_{q-1} \cdot d_{q-1}-m_{q-1} \cdot d_{q} & \text { if } q \geq 2 \\
0 & \text { if } q=1
\end{array}\right.
$$

Let $Z$ be an indeterminate and define a $(q, Z)$ deformation of $y(\underline{t})$ to by any $y^{*}(Z, \underline{t}) \in \mathbf{K}(Z)[[\underline{t}]]$ such that

$$
\operatorname{In}\left(y^{*}(Z, \underline{t})-\sum_{p \in M_{q}} c_{p} \cdot \underline{t}^{p}\right)=Z \cdot \underline{t}^{m_{q}}
$$


Equivalently a $(q, Z)$-deformation $y^{*}(Z, \underline{t})$ of $y(\underline{t})$ is any element $y^{*}(Z, \underline{t}) \in \mathbf{K}(Z)[[\underline{t}]]$ such that:

$$
y^{*}(Z, \underline{t})=y(\underline{t})+\left(Z-c_{m_{q}}\right) \cdot \underline{t}^{m_{q}}+u(Z, \underline{t})
$$

where $O_{\underline{t}}(u(Z, \underline{t}))>\left|m_{q}\right|$. Let $z(\underline{t}) \in \operatorname{Root}(f)$ and let $y^{*}(Z, \underline{t})$ be a $(q, Z)$ deformation of $y(\underline{t})$. We want to calculate the contact between $y^{*}(Z, \underline{t})$ and $z(\underline{t})$. Note that:

$$
y^{*}(Z, \underline{t})-z(\underline{t})=\left(Z-c_{m_{q}}\right) \cdot \underline{t}^{m_{q}}+y(\underline{t})-z(\underline{t})+u(\underline{t}, Z)
$$

It follows that if $z(\underline{t}) \in Q(q)$, then $\operatorname{In}\left(y^{*}(Z, \underline{t})-z(\underline{t})\right)=\operatorname{In}(y(\underline{t})-z(\underline{t}))$. In particular:

$$
\text { (1) In } \prod_{z(\underline{\underline{t}}) \in Q(q)}\left(y^{*}(Z, \underline{t})-z(\underline{t})\right)= \begin{cases}a_{1} & \text { if } q=1 \\ a_{q} t^{r_{q-1} \cdot d_{q-1}-m_{q-1} d_{q}} & \text { if } q \geq 2\end{cases}
$$

where for all $q \geq 1, a_{q}$ is a nonzero constant of $\mathbf{K}$. On the other hand, if $z(\underline{t}) \in R(q)$, then $\exp (z(\underline{t})-y(\underline{t})) \geq m_{q}$, then $\operatorname{Inco}\left(y^{*}(Z, \underline{t})-z(\underline{t})\right)=\left(Z-c_{m_{q}}\right)+\left(c_{m_{q}}-w(z) c_{m_{q}}\right)=Z-w(z) c_{m_{q}}$, in particular $\operatorname{In}\left(y^{*}(Z, \underline{t})-z(\underline{t})\right)=$ $\left(Z-w(z) c_{m_{q}}\right) \underline{t}^{m_{q}}$. Consequently

$$
\operatorname{In}\left(\prod_{z(\underline{t}) \in R(q)}\left(y^{*}(Z, \underline{t})-z(\underline{t})\right)\right)=\left(Z^{e_{q}}-c_{m_{q}}^{e_{q}}\right)^{d_{q+1}} \cdot \underline{t}^{m_{q} \cdot d_{q}} .
$$

Now

$$
f\left(t_{1}^{n}, \ldots, t_{e}^{n}, y^{*}(Z, \underline{t})\right)=\prod_{z(\underline{t}) \in Q(q)}\left(y^{*}(Z, \underline{t})-z(\underline{t})\right) . \prod_{z(\underline{t}) \in R(q)}\left(y^{*}(Z, \underline{t})-z(\underline{t})\right)
$$

and $r_{q} d_{q}=r_{q-1} d_{q-1}+m_{q} d_{q}-m_{q-1} d_{q}$, in particular:

$$
\operatorname{In}\left(f\left(t_{1}^{n}, \ldots, t_{e}^{n}, y^{*}\right)\right)=\alpha\left(Z^{e_{q}}-y_{m_{q}}^{e_{q}}\right)^{d_{q+1}} \cdot \underline{t}^{r_{q} \cdot d_{q}} .
$$

where $\alpha \in \mathbf{K}^{*}$.

Lemma 4.1 Let $q \in \mathbf{N}, 1 \leq q \leq h$. Let $F=F(\underline{x}, y) \in \mathbf{R}[y]$ such that $\operatorname{deg}_{y} F<\frac{n}{d_{q}}$. Let $y^{*}(Z, \underline{t})$ be a $(q, Z)$-deformation of $y(\underline{t})$. Then $\operatorname{inco}\left(F\left(t_{1}^{n}, \ldots, t_{e}^{n}, y^{*}(Z, \underline{t})\right)\right) \in \mathbf{K}^{*}$.

Proof. If $q=1$, then $\operatorname{deg}_{y}(F)=0$, in particular $F(\underline{x}, y) \in \mathbf{R}$, and $\left.F\left(t_{1}^{n}, \ldots, t_{e}^{n}, y^{*}(Z, \underline{t})\right) \in \mathbf{K}[[\underline{t}]]\right)$. Let $q \geq 2$ and for all $1 \leq k<q$, let $G_{k}(\underline{x}, y)$ be a pseudo $d_{k}$ th root of $f$. Let $G^{q}=\left(G_{1}, \ldots, G_{q-1}\right)$ and let $B\left(G^{q}\right)=\left\{\left(\theta_{1}, \ldots, \theta_{q-1}\right) ; 0 \leq \theta_{k}<e_{k}\right.$ for all $\left.1 \leq k<q\right\}$. Let:

$$
F=\sum_{\underline{\theta} \in B\left(G^{q}\right)} c_{\underline{\theta}}(\underline{x}) \cdot G_{1}^{\theta_{1}} \ldots \ldots G_{q-1}^{\theta_{q-1}}
$$

be the $G^{q}$-adic expansion of $F$. Since $O\left(f, G_{k}\right)=\exp \left(G_{k}\left(t_{1}^{n}, \ldots, t_{e}^{n}, y(\underline{t})\right)\right)=r_{k}$ and $c\left(f, G_{k}\right)=m_{k}<m_{q}$, then $\exp \left(G_{k}\left(t_{1}^{n}, \ldots, t_{e}^{n}, y^{*}(Z, \underline{t})\right)\right)=r_{k}$. In particular there is a unique $\underline{\theta}^{0} \in B\left(G^{q}\right)$ such that:

$$
\exp \left(F\left(t_{1}^{n}, \ldots, t_{e}^{n}, y^{*}(Z, \underline{t})\right)\right)=\exp \left(c_{\underline{\theta}^{0}}\left(t_{1}^{n}, \ldots, t_{e}^{n}\right)\right)+\sum_{k=1}^{q-1} \theta_{k}^{0} \cdot \exp \left(G_{k}\left(t_{1}^{n}, \ldots, t_{e}^{n}, y^{*}(Z, \underline{t})\right)\right)
$$




$$
=\exp \left(c_{\underline{\theta}_{0}}\left(t_{1}^{n}, \ldots, t_{e}^{n}\right)\right)+\sum_{k=1}^{q-1} \theta_{k}^{0} r_{k}
$$

In particular:

$$
\operatorname{In}\left(F\left(t_{1}^{n}, \ldots, t_{e}^{n}, y^{*}(Z, \underline{t})\right)\right)=\operatorname{In}\left(c_{\underline{\theta}^{0}}\left(t_{1}^{n}, \ldots, t_{e}^{n}\right) \cdot\left(G_{1}^{\theta_{1}} \ldots \ldots G_{q-1}^{\theta_{q-1}}\right)\left(t_{1}^{n}, \ldots, t_{e}^{n}, y^{*}(Z, \underline{t})\right)\right) .
$$

But inco $\left(\left(c_{\underline{\theta}^{0}}\left(t_{1}^{n}, \ldots, t_{e}^{n}\right)\right) \in \mathbf{K}^{*}\right.$ and by $(1)$, inco $\left(g_{k}\left(t_{1}^{n}, \ldots, t_{e}^{n}, y^{*}(Z, \underline{t})\right) \in \mathbf{K}^{*}\right.$ for all $1 \leq k \leq q-1$. This implies our assertion.

Lemma 4.2 Let $q \in \mathbf{N}, 2 \leq q \leq h$ and let $g=g(\underline{x}, y) \in \mathbf{R}[y]$ be a monic polynomial of degree $\frac{n}{d_{q}}$ in $y$. Let $y^{*}(Z, \underline{t})$ be a $(q, Z)$-deformation of $y(t)$. If $\operatorname{In}\left(g\left(t_{1}^{n}, \ldots, t_{e}^{n}, y^{*}(Z, \underline{t})\right)\right)=\alpha . Z t^{r_{q}}, \alpha \in \mathbf{K}^{*}$, then $\operatorname{In}\left(\left(\tau_{f} g\right)\left(t_{1}^{n}, \ldots, t_{e}^{n}, y^{*}(Z, \underline{t})\right)\right)=\alpha \cdot Z t^{r_{q}}$.

Proof. Let

$$
f=g^{d_{q}}+a_{1} g^{d_{q}-1}+\ldots+a_{d_{q}}
$$

be the $g$-adic expansion of $f$, and recall that $\tau_{f}(g)=g+d_{q}^{-1} a_{1}$. We need to show that $r_{q}<\exp \left(a_{1}\left(t_{1}^{n}, \ldots, t_{e}^{n}, y^{*}(Z, \underline{t})\right)\right)$. We have

$$
f\left(t_{1}^{n}, \ldots, t_{e}^{n}, y^{*}(Z, \underline{t})\right)=\sum_{k=0}^{d_{q}} a_{k}\left(t_{1}^{n}, \ldots, t_{e}^{n}, y^{*}(Z, \underline{t})\right) \cdot g^{d_{q}-k}\left(t_{1}^{n}, \ldots, t_{e}^{n}, y^{*}(Z, \underline{t})\right)
$$

where $a_{0}=1$. Let

$$
u=\inf \left\{\exp \left(a_{k}\left(t_{1}^{n}, \ldots, t_{e}^{n}, y^{*}(Z, \underline{t})\right) \cdot g^{d_{q}-k}\left(t_{1}^{n}, \ldots, t_{e}^{n}, y^{*}(Z, \underline{t})\right)\right) ; 0 \leq k \leq d_{q}\right\} .
$$

Since $a_{0}=1$ and $\exp \left(g^{d_{q}}\left(t_{1}^{n}, \ldots, t_{e}^{n}, y^{*}(Z, \underline{t})\right)\right)=d_{q} \cdot r_{q}$, then $u \in \mathbf{N}^{e}$. Let

$$
I=\left\{0 \leq k \leq d_{q} ; \exp \left(a_{k}\left(t_{1}^{n}, \ldots, t_{e}^{n}, y^{*}(\underline{t}, Z)\right) \cdot g^{d_{q}-k}\left(t_{1}^{n}, \ldots, t_{e}^{n}, y^{*}(\underline{t}, Z)\right)\right)=u\right\} .
$$

then for all $k \in I, a_{k}\left(t_{1}^{n}, \ldots, t_{e}^{n}, y^{*}(Z, \underline{t})\right) \neq 0$ and, by lemma 4.1., inco $\left(a_{k}\left(t_{1}^{n}, \ldots, t_{e}^{n}, y^{*}(Z, \underline{t})\right)\right)=\alpha_{k} \in \mathbf{K}^{*}$. Consequently inco $\left(a_{k}\left(t_{1}^{n}, \ldots, t_{e}^{n}, y^{*}(Z, \underline{t})\right) \cdot g^{d_{q}-k}\left(t_{1}^{n}, \ldots, t_{e}^{n}, y^{*}(Z, \underline{t})\right)\right)=\alpha_{k} \cdot \alpha^{d_{q}-k} \cdot Z^{d_{q}-k}$ for all $k \in I$. In particular $\operatorname{In}\left(f\left(t_{1}^{n}, \ldots, t_{e}^{n}, y^{*}(Z, \underline{t})\right)\right)=\left(\sum_{k \in I} \alpha_{k} \cdot \alpha^{d_{q}-k} \cdot Z^{d_{q}-k}\right) \cdot t^{u}$. But

$$
\operatorname{In}\left(f\left(t_{1}^{n}, \ldots, t_{e}^{n}, y^{*}(\underline{t}, Z)\right)\right)=a\left(Z^{e_{q}}-y_{m_{q}}^{e_{q}}\right)^{d_{q+1}} \cdot t^{r_{q} \cdot d_{q}}, a \in \mathbf{K}^{*}
$$

so:

$$
u=r_{q} \cdot d_{q} \quad \text { and } \quad \sum_{k \in I} \alpha_{k} \cdot \alpha^{d_{q}-k} \cdot Z^{d_{q}-k}=a\left(Z^{e_{q}}-y_{m_{q}}^{e_{q}}\right)^{d_{q+1}}
$$

in particular $\sum_{k \in I} \alpha_{k} \cdot \alpha^{d_{q}-k} \cdot Z^{d_{q}-k} \in \mathbf{K}\left[Z^{e_{q}}\right]$. On the other hand $e_{q}=\frac{d_{q}}{d_{q+1}}$ doesn't divide $d_{q}-1$, then $d_{q}-1 \notin I$, so $u<\exp \left(a_{1}\left(t_{1}^{n}, \ldots, t_{e}^{n}, y^{*}(Z, \underline{t})\right) \cdot g^{d_{q}-1}\left(t_{1}^{n}, \ldots, t_{e}^{n}, y^{*}(Z, \underline{t})\right)\right)=\exp \left(a_{1}\left(t_{1}^{n}, \ldots, t_{e}^{n}, y^{*}(Z, \underline{t})\right)\right)+\left(d_{q}-1\right) \cdot r_{q}$. This proves our assertion.

As a corollary we get the following theorem: 
Theorem 4.3 Let the notations be as above, and let $d_{1}, \ldots, d_{h}, d_{h+1}=1$ be the gcd-sequence of $f$. Then $O\left(f, \operatorname{App}_{d_{k}}(f)\right)=r_{k}$ for all $1 \leq k \leq h$.

Proof. For all $1 \leq k \leq h$, let $G_{k}$ be a pseudo $d_{k}$ th root of $f$. Then $\operatorname{deg}_{y}\left(G_{k}\right)=\frac{n}{d_{k}}$. But $\operatorname{App}_{d_{k}}(f)=\tau_{f}\left(G_{k}\right)$. Now use Lemma 4.2.

\section{$5 \quad$ Generalized Newton polygons}

Let $n \in \mathbf{N}$ and let $\underline{r}_{0}=\left(r_{0}^{1}, \ldots, r_{0}^{e}\right)$ be the canonical basis of $(n \mathbf{Z})^{e}$. Let $r_{1}<\ldots<r_{h}$ be a sequence of elements of $\mathbf{N}^{e}$, where $<$ means $<$ coordinate-wise. Set $D_{1}=n^{e}$ and for all $1 \leq k \leq h$, let $D_{k+1}$ be the GCD of the $(e, e)$ minors of the $(e, e+k)$ matrix $\left(n . I(e, e),\left(r_{1}\right)^{T}, \ldots,\left(r_{k}\right)^{T}\right)$. Suppose that $n^{e-1}$ divides $D_{k}$ for all $1 \leq k \leq h+1$ and that $D_{h+1}=n^{e-1}$, and also that $D_{1}>D_{1}>\ldots>D_{h+1}$, in such a way that if we set $d_{1}=n$ and $d_{k}=\frac{D_{k}}{n^{e-1}}$ for all $2 \leq k \leq h$, then $d_{1}=n>d_{2}>\ldots>d_{h+1}=1$.

For all $1 \leq k \leq h$, let $g_{k}$ be a monic polynomial of degree $\frac{n}{d_{k}}$ in $y$ and set $G=\left(g_{1}, \ldots, g_{h}\right)$. Let $F$ be a nonzero polynomial of $\mathbf{K}[[\underline{x}]][y]$ and let:

$$
F=\sum_{\underline{\theta} \in B(G)} c_{\underline{\theta}}(\underline{x}) g_{1}^{\theta_{1}} \ldots . . g_{h}^{\theta_{h}}
$$

where $B(G)=\left\{\underline{\theta}=\left(\theta_{1}, \ldots, \theta_{h}\right) ; \forall 1 \leq i \leq h-1,0 \leq \theta_{i}<e_{i}=\frac{d_{i}}{d_{i+1}}\right.$ and $\left.\theta_{h}<+\infty\right\}$, be the $G$-adic expansion of $F$. Let $\operatorname{Supp}_{G}(F)=\left\{\underline{\theta} \in B(G) ; c_{\underline{\theta}} \neq 0\right\}$. If $\theta \in \operatorname{Supp}_{G}(F)$ and $\underline{\gamma}=\exp \left(c_{\underline{\theta}}(\underline{x})\right)$, we shall associate with the monomial $c_{\underline{\theta}}(\underline{x}) g_{1}^{\theta_{1}} \ldots . g_{h}^{\theta_{h}}$ the $e$-uplet

$$
<\left((\underline{\gamma}, \underline{\theta}),\left(\underline{r}_{0}, \underline{r}\right)\right)>=\sum_{i=1}^{e} \gamma_{i} \cdot r_{0}^{i}+\sum_{j=1}^{h} \theta_{j} \cdot r_{j}
$$

There is a unique $\underline{\theta}^{0} \in \operatorname{Supp}_{G}(F)$ such that if $\underline{\gamma}^{0}=\exp \left(c_{\underline{\theta}^{0}}(\underline{x})\right)$, then:

$$
<\left(\left(\underline{\gamma}^{0}, \underline{\theta}^{0}\right),\left(\underline{r}_{0}, \underline{r}\right)\right)>=\inf \left\{<\left((\gamma, \underline{\theta}),\left(r_{0}, \underline{r}\right)\right)>, \underline{\theta} \in \operatorname{Supp}_{G}(F)\right\}
$$

We set

$$
\mathrm{fO}(\underline{r}, G, F)=<\left(\left(\underline{\gamma}^{0}, \underline{\theta}^{0}\right),\left(\underline{r}_{0}, \underline{r}\right)\right)>
$$

and we call it the formal order of $F$ with respect to $(\underline{r}, G)$. We also set:

$$
M_{G}(F)=M\left(c_{\underline{\theta}_{0}}\right) \cdot g_{1}^{\theta_{1}^{0}} \ldots . g_{h}^{\theta_{h}^{0}}
$$

and we call it the initial monomial of $F$ with respect to $(\underline{r}, G)$.

Let $f=y^{n}+a_{1}(\underline{x}) y^{n-1}+\ldots+a_{n}(\underline{x})$ be a quasi-ordinary polynomial of $\mathbf{K}\left[\left[x_{1}, \ldots, x_{e}\right]\right][y]$ and let $d \in \mathbf{N}$ be a divisor of $n$. Let $g$ be a monic polynomial of $\mathbf{K}\left[\left[x_{1}, \ldots, x_{e}\right]\right][y]$ of degree $\frac{n}{d}$ in $y$ and let:

$$
f=g^{d}+a_{1}(\underline{x}, y) g^{d-1}+\ldots+a_{d}(\underline{x}, y)
$$


be the $g$-adic expansion of $f$. We associate with $f$ the set of points:

$$
\left\{\left(\mathrm{fO}\left(\underline{r}, G, a_{k}\right),(d-k) \mathrm{fO}(\underline{r}, G, g)\right), k=0, \ldots, d\right\} \subseteq \mathbf{N}^{e} \times \mathbf{N}^{e}
$$

We denote this set by $\operatorname{GNP}(f, \underline{r}, G, g)$ and we call it the generalized Newton polygon of $f$ with respect to $(\underline{r}, G, g)$. Note that if $e=1$ and $f$ is an irreducible polynomial of $\mathbf{K}[[x]][y]$, then the above set is equivalent to the usual Newton polygon of $f$.

Definition 5.1 We say that $f$ is straight with respect to $(\underline{r}, G, g)$ if the following holds:

i) $\mathrm{fO}\left(\left(\underline{r}, G, a_{d}\right)=d . \mathrm{fO}((\underline{r}, G, g))\right.$.

ii) For all $1 \leq k \leq h-1, \mathrm{fO}\left(\underline{r}, G, a_{k}\right) \geq k \cdot \mathrm{fO}((\underline{r}, G, g))$, where $\geq$ mean $\geq$ coordinate-wise.

We say that $f$ is strictly straight with respect to $(\underline{r}, G, g)$ if the inequality in ii) is a strict inequality.

\section{The criterion}

Let $f=y^{n}+a_{1}(x) y^{n-1}+\ldots+a_{n}(x)$ be a nonzero element of $\mathbf{K}\left[\left[x_{1}, \ldots, x_{e}\right]\right][y]$ and assume, after an eventual change of variables, that $a_{1}(\underline{x})=0$. Let $\underline{r}_{0}=\left(r_{0}^{1}, \ldots, r_{0}^{e}\right)$ be the canonical basis of $(n \mathbf{Z})^{e}$ and let $d_{1}=n$. Let $g_{1}=y$ be the $d_{1}$-th approximate root of $f$ and set $m_{1}=r_{1}=\exp \left(a_{n}(\underline{x})\right)$. Let $D_{2}$ be the gcd of the $(e, e)$ minors of the $(e, e+1)$ matrix $\left(n . I(e, e), m_{1}^{T}\right)$. Let $d_{2}=\frac{D_{2}}{n^{e-1}}$ and let $g_{2}$ be the $d_{2}$-th approximate root of $f$ and set $e_{2}=\frac{d_{1}}{d_{2}}=\frac{n}{d_{2}} \ldots$. Suppose that we constructed $\left(r_{1}, \ldots, r_{k-1}\right),\left(m_{1}, \ldots, m_{k-1}\right)$, and $\left(d_{1}, \ldots, d_{k}\right)$, then let $g_{k}$ be the $d_{k}$-th approximate root of $f$ and let

$$
f=g_{k}^{d_{k}}+\beta_{2}^{k} g_{k}^{d_{k}-2}+\ldots+\beta_{d_{k}}^{k}
$$

be the $g_{k}$-adic expansion of $f$. Then $r_{k}=\mathrm{fO}\left(\underline{r}^{k}, G^{k}, \beta_{d_{k}}^{k}\right)$, where $\underline{r}^{k}=\left(\frac{r_{0}^{1}}{d_{k}}, \ldots, \frac{r_{0}^{e}}{d_{k}}, \frac{r_{1}}{d_{k}}, \ldots, \frac{r_{k-1}}{d_{k}}\right)$ and $G^{k}=$ $\left(g_{1}, \ldots, g_{k-1}\right)$. With these notations we have the following:

Theorem 6.1 The polynomial $f$ is an irreducible quasi-ordinary polynomial if and only if the following holds:

i) There is an integer $h$ such that $d_{h+1}=1$.

ii) For all $1 \leq k \leq h-1, r_{k} d_{k}<r_{k+1} d_{k+1}$, where $<$ means $<$ coordinate-wise.

iii) For all $2 \leq k \leq h+1, g_{k}$ is strictly straight with respect to $\left(\underline{r}^{k}, G^{k}, g_{k-1}\right)$.

We shall first prove the following results:

Lemma 6.2 Let $c \in \mathbf{K}^{*}$. The quasi-ordinary polynomial $F=y^{n}-c x_{1}^{\alpha_{1}} \ldots \ldots x_{e}^{\alpha_{e}}$ is irreducible in $\mathbf{K}\left[\left[x_{1}, \ldots, x_{e}\right]\right][y]$ if and only if $\operatorname{gcd}\left(n, \alpha_{1}, \ldots, \alpha_{e}\right)=1$, or equivalently if and only if the gcd of the $(e, e)$ minors of the matrix $\left(n I(e, e),\left(\alpha_{1}, \ldots, \alpha_{e}\right)^{T}\right)$ is $n^{e-1}$.

Proof. Let $\tilde{c}$ be an n-th root of $c$ in $\mathbf{K}$ and let $Y=\tilde{c} x_{1}^{\frac{\alpha_{1}}{n}} \ldots \ldots x_{e^{\frac{\alpha_{e}}{n}}}^{\in} \mathbf{K}\left(\left(x_{1}^{\frac{1}{n}}, \ldots, x_{e}^{\frac{1}{n}}\right)\right)$. Then $F$ is the minimal polynomial of $Y$ over $\mathbf{K}\left(\left(x_{1}, \ldots, x_{e}\right)\right)$. In particular it is irreducible.

Proposition 6.3 Assume that the polynomial $f$ is irreducible and let $\left(m_{k}\right)_{1 \leq k \leq h}$ be the set of characteristic exponents of $f$. Let $F$ be a quasi-ordinary polynomial of $\mathbf{K}\left[\left[x_{1}, \ldots, x_{e}\right]\right][y]$ and assume that $F$ is monic of degree $n$ in $y$. If $O(f, F)>r_{h} d_{h}$, then $F$ is irreducible in $\mathbf{K}\left[\left[x_{1}, \ldots, x_{e}\right]\right][y]$. 
Proof. Assume that $F$ is not irreducible and let $\tilde{F}$ be an irreducible component of $F$ in $\mathbf{K}\left[\left[x_{1}, \ldots, x_{e}\right]\right][y]$. Let $C=c(f, \tilde{F})$ be the contact of $f$ with $\tilde{F}$. If $C \in M_{h+1}$ and $C \neq m_{h}$, then $\operatorname{deg}_{y}(\tilde{F}) \geq n$, which is a contradiction because $F$ is not irreducible. In particular, $O(f, \tilde{F}) \leq r_{h} d_{h} \cdot \frac{\operatorname{deg}_{y}(\tilde{F})}{n}$. Since this is true for all irreducible component of $F$, then $O(f, F) \leq r_{h} d_{h} \cdot \frac{\operatorname{deg}_{y}(F)}{n}=r_{h} d_{h}$, which is a contradiction.

Proof of Theorem 6.1.. Suppose first that $f$ is irreducible. Then the condition i) is obvious. On the other hand, if we denote by $\left(m_{k}\right)_{1 \leq k \leq h}$ the set of characteristic exponents of $f$, then

$$
r_{k+1} d_{k+1}=r_{k} d_{k}+\left(m_{k+1}-m_{k}\right) \cdot d_{k+1}
$$

for all $1 \leq k \leq h-1$. This proves ii). Now for all $1 \leq k \leq h+1, g_{k}$ is an irreducible quasi-ordinary polynomial and $g_{1}, \ldots, g_{k-1}$ are the approximate roots of $g_{k}$. In particular, to prove iii), it suffices to prove that $f=g_{h+1}$ is straight with respect to $\left(\underline{r}, G, g_{h}\right)=\left(\underline{r}^{h+1}, G^{h+1}, g_{h}\right)$. Let

$$
f=g_{h}^{d_{h}}+\beta_{2}^{h} g_{h}^{d_{h}-2}+\ldots+\beta_{d_{h}}^{h}
$$

be the $g_{h}$-adic expansion of $f$. If we denote by $\Gamma^{h}$ the semigroup generated by $r_{1}^{0}, \ldots, r_{e}^{0}, r_{1}, \ldots, r_{h-1}$, then we have the following:

- For all $2 \leq i \leq h-1, O\left(\beta_{i}^{h}, f\right) \in \Gamma^{h}$.

- For all $0<a<d_{h}, a . r_{h} \notin \Gamma^{h}$.

It follows that for all $2 \leq i \leq h-1, O\left(\beta_{i}^{h}, f\right) \neq i . r_{h}$ and for all $2 \leq i \neq j \leq d_{h}-1, O\left(\beta_{i}^{h}, f\right)+\left(d_{h}-i\right) r_{h} \neq$ $O\left(\beta_{j}^{h}, f\right)+\left(d_{h}-j\right) r_{h}$. Since $O\left(g_{h}^{d_{h}}, f\right)=r_{h} d_{h}$, then $O\left(\beta_{d_{h}}^{h}, f\right)=r_{h} d_{h}$ and $O\left(\beta_{i}^{h}, f\right)>i r_{h}$ for all $2 \leq i \leq d_{h}-1$. This implies iii).

Conversely suppose that $f$ verifies the conditions i), ii), and iii). We shall prove by induction on $h$ that $f$ is irreducible. Suppose first that $h=1$, then $f=y^{n}+a_{2}(\underline{x}) y^{n-2}+\ldots+a_{n}(\underline{x})$ and $O_{x}\left(a_{i}(\underline{x})\right)>i . O_{x}\left(a_{n}(\underline{x})\right)$ for all $2 \leq i \leq n-1$. Furthermore, $D_{2}=n^{e-1}$. In particular $F=y^{n}+M\left(a_{1}(\underline{x})\right)$ is irreducible by Lemma 6.2. But $O(F, f)=O(f-F, f)>r_{1} d_{1}$, then $f$ is irreducible by Proposition 6.3.

Let $h>1$ and assume that $g_{k}$ is an irreducible quasi-ordinary polynomial for all $1 \leq k \leq h$. Let

$$
f=g_{h}^{d_{h}}+\beta_{2}^{h} g_{h}^{d_{h}-2}+\ldots+\beta_{d_{h}}^{h}
$$

be the $g_{h}$-adic expansion of $f$ and let $F=g_{h}^{d_{h}}+M_{G^{h}}\left(\beta_{d_{h}}^{h}\right)$. We shall prove that $F$ is irreducible. Let to this end $Y(\underline{t})=\sum_{p} Y(p) \underline{t}^{p}$ be a root of $g_{h}\left(t_{1}^{\frac{n}{d_{h}}}, \ldots, t_{e}^{\frac{n}{d_{h}}}, y\right)=0$ and consider the $\left(\frac{m_{h}}{d_{h}}, Z\right)$ deformation $\tilde{Y}=\sum_{p \in \frac{1}{d_{h}} \cdot M_{h}} Y(p) \underline{t}^{p}+Z t^{\frac{m_{h}}{d_{h}}}$ of $Y(\underline{t})$. Let $M_{G^{h}}\left(\beta_{d_{h}}^{h}\right)=c \cdot \underline{x}^{\theta_{0}} \cdot g_{1}^{\theta_{1}} \ldots \ldots g_{h-1}^{\theta_{h-1}}$, where $c \in \mathbf{K}^{*}$. Since $g_{h}$ is irreducible, then

$$
O\left(F, g_{h}\right)=O\left(M_{G^{h}}\left(\beta_{d_{h}}^{h}\right), g_{h}\right)=\sum_{i=1}^{e} \theta_{0}^{i} \frac{r_{i}^{0}}{d_{h}}+\sum_{k=1}^{h-1} \theta_{k} \frac{r_{k}}{d_{h}}
$$

but $g_{h}\left(t_{1}^{\frac{n}{d_{h}}}, \ldots, t_{e}^{\frac{n}{d_{h}}}, \tilde{Y}\right)=c(Z) t^{\frac{r_{h}}{d_{h}}}, \operatorname{deg}_{Z} c(Z)>0$, and $\operatorname{inco}\left(g_{k}\left(t_{1}^{\frac{n}{d_{h}}}, \ldots, t_{e}^{\frac{n}{d_{h}}}, \tilde{Y}\right)\right) \in \mathbf{K}^{*}$ for all $1 \leq k \leq h-1$, in particular $\operatorname{info}\left(F\left(t_{1}^{n}, \ldots, t_{e}^{n}, \tilde{Y}\left(t_{1}^{d_{h}}, \ldots, t_{e}^{d_{h}}, Z\right)\right)=\tilde{c}(Z) t^{r_{h}}\right.$ and $\operatorname{deg}_{Z}(\tilde{c}(Z))>0$. This implies that there exists $z_{0} \in \mathbf{K}$ such that if $y(\underline{t})=\tilde{Y}\left(t_{1}^{d_{h}}, \ldots, t_{e}^{d_{h}}, z_{0}\right)$, then $\exp \left(F\left(t_{1}^{n}, \ldots, t_{e}^{n}, y(\underline{t})\right)\right)>r_{h} d_{h}$. Since $F$ is monic in $y$ and the minimal polynomial of $y\left(x_{1}^{\frac{1}{n}}, \ldots, x_{e}^{\frac{1}{n}}\right)$ over $\mathbf{K}\left(\left(x_{1}, \ldots, x_{e}\right)\right)$ is of degree $n$, then this polynomial coincides with 
$F$, which is consequently irreducible. Now $O(F, f)=O(F-f, f)>r_{h} d_{h}$, then $f$ is irreducible by Proposition 6.3 .

\section{$7 \quad$ Examples}

Example 1: Let $f=y^{8}-2 x_{1} x_{2} y^{4}+x_{1}^{2} x_{2}^{2}-x_{1}^{3} x_{2}^{2} \in \mathbf{K}\left[\left[x_{1}, x_{2}\right]\right][y]$. Then we have:

- $D_{1}=n^{2}=8^{2}=64, d_{1}=n=8, r_{0}^{1}=(8,0), r_{0}^{2}=(0,8), g_{1}=\operatorname{App}_{d_{1}}(f)=y$, and $r_{1}=O\left(f, g_{1}\right)=(2,2)$.

- $D_{2}$ is the gcd of the $(2,2)$ minors of the matrix $\left(8 . I(2,2),(2,2)^{T}\right)$, then $D_{2}=16=8.2$, in particular $d_{2}=2$. Since $f=\left(y^{4}-x_{1} x_{2}\right)^{2}-x_{1}^{3} x_{2}^{2}$, then $g_{2}=\operatorname{App}_{d_{2}}(f)=y^{4}-x_{1} x_{2}$. Let $\underline{r}^{2}=\left(\frac{r_{0}^{1}}{d_{2}}, \frac{r_{0}^{2}}{d_{2}}, \frac{r_{1}}{d_{2}}\right)=((4,0),(0,4),(1,1))$ and $\underline{G}^{2}=\left(g_{1}\right)$, then $r_{2}=\mathrm{fO}\left(\underline{r}^{2}, \underline{G}^{2}, x_{1}^{3} x_{2}^{2}\right)=3(4,0)+2(0,4)=(12,8)$.

- $D_{3}$ is the gcd of the $(2,2)$ minors of the matrix $\left(8 \cdot I(2,2),(2,2)^{T},(12,8)^{T}\right)$, then $D_{3}=8$, in particular $d_{3}=1$.

- Now $\operatorname{GNP}\left(g_{2}, \underline{r}^{2}, \underline{G}^{2}\right)=\{((0,0), 4 .(1,1)),((4,4),(0,0))\}$ and $\operatorname{GNP}\left(f, \underline{r}^{3}=\left(r_{0}^{1}, r_{0}^{2}, r_{1}, r_{2}\right), \underline{G}^{3}=\left(g_{1}, g_{2}\right)\right)=$ $\{((0,0), 2 .(12,8)),((24,16),(0,0))\}$, then the strict straightness condition is verified. Since $r_{1} d_{1}<r_{2} d_{2}$, then $f$ is irreducible. Note that $m_{2}=(10,6)$ is the second characteristic exponent of $f$.

Example 2: Let $f=y^{8}-2 x_{1} x_{2} y^{4}+x_{1}^{2} x_{2}^{2}-x_{1}^{4} x_{2}^{2}-x_{1}^{5} x_{2}^{3} \in \mathbf{K}\left[\left[x_{1}, x_{2}\right]\right][y]$. Then we have:

- $D_{1}=n^{2}=8^{2}=64, d_{1}=n=8, r_{0}^{1}=(8,0), r_{0}^{2}=(0,8), g_{1}=\operatorname{App}_{d_{1}}(f)=y$, and $r_{1}=O\left(f, g_{1}\right)=(2,2)$.

- $D_{2}$ is the ged of the $(2,2)$ minors of the matrix $\left(8 \cdot I(2,2),(2,2)^{T}\right)$, then $D_{2}=16=8.2$, in particular $d_{2}=2$.

Since $f=\left(y^{4}-x_{1} x_{2}\right)^{2}-x_{1}^{4} x_{2}^{2}-x_{1}^{5} x_{2}^{3}$, then $g_{2}=\operatorname{App}_{d_{2}}(f)=y^{4}-x_{1} x_{2}$. Let $\underline{r}^{2}=\left(\frac{r_{0}^{1}}{d_{2}}, \frac{r_{0}^{2}}{d_{2}}, \frac{r_{1}}{d_{2}}\right)=((4,0),(0,4),(1,1))$ and $\underline{G}^{2}=\left(g_{1}\right)$, then $r_{2}=\mathrm{fO}\left(\underline{r}^{2}, \underline{G}^{2}, x_{1}^{4} x_{2}^{2}\right)=4(4,0)+2(0,4)=(16,8)$.

- $D_{3}$ is the gcd of the $(2,2)$ minors of the matrix $\left(8 . I(2,2),(2,2)^{T},(16,8)^{T}\right)$, then $D_{3}=16$, in particular $d_{3}=d_{2}=2$. In particular $f$ is not irreducible. Note that in this example the strict straightness condition is verified for $f$ and $g_{2}$.

Example 3: Let $f=y^{8}-2 x_{1} x_{2} y^{4}+x_{1}^{3} x_{2}^{2}-x_{1} y^{5} \in \mathbf{K}\left[\left[x_{1}, x_{2}\right]\right][y]$. Then we have:

- $D_{1}=n^{2}=8^{2}=64, d_{1}=n=8, r_{0}^{1}=(8,0), r_{0}^{2}=(0,8), g_{1}=\operatorname{App}_{d_{1}}(f)=y$, and $r_{1}=O\left(f, g_{1}\right)=(3,2)$.

- $D_{2}$ is the gcd of the $(2,2)$ minors of the matrix $\left(8 . I(2,2),(3,2)^{T}\right)$, then $D_{2}=8$, in particular $d_{2}=1$.

- $\operatorname{GNP}\left(f, \underline{r}^{2}=\left(r_{0}^{1}, r_{0}^{2}, r_{1}\right), \underline{G}^{2}=\left(g_{1}\right)\right)=\{((0,0), 8 .(3,2)),((8,0), 5 .(3,2)),((8,0)+(0,8), 4 .(3,2)),(3 .(8,0)+$ $2 .(0,8),(0,0))\}=\{((0,0),(24,16)),((8,0),(15,10)),((8,8),(12,8)),((24,16),(0,0))\}$. Here the strict straightness is not verified, then $f$ is not irreducible.

\section{References}

[1] S.S. Abhyankar.- Expansion Techniques in Algebraic Geometry, Lecture Notes of the Tata Institute Bombay, $57,(1977)$.

[2] S.S. Abhyankar.- On the ramification of algebraic functions, Amer. J. Math. 77 (1955), 575-592.

[3] S.S. Abhyankar.-Irreducibility criterion for germs of analytic functions of two complex variables. Adv. Math., $74 \mathrm{n}^{\circ} 2(1989), 190-257$.

[4] P.D. Gonzalez Perez.- The semigroup of a quasi-ordinary hypersurface, Journal of the Institute of Mathematics of Jussieu, $\mathrm{n}^{\circ} 2$ (2003), 383-399. 
[5] K. Kiyek and M. Micus.- Semigroup of a quasiordinary singularity, Banach Center Publications, Topics in Algebra, Vol. 26 (1990), 149-156.

[6] Lejeune-Jalabert Monique.- Sur l'équivalence des singularités des courbes algebroides planes. Coefficients de Newton, Publications de l'Ecole Polytechnique (1969).

[7] J.Lipman.- Quasi-ordinary singularities of embedded surfaces, Thesis, Harvard University (1965).

[8] P. Popescu-Pampu.- Arbres de contact des singularités quasi-ordinaires et graphes d'adjacence pour les 3-variétés réelles, Thèse de doctorat de l'universite de Paris 7 (2001). 